\title{
LE CORPS DANS LAUTISME SELON JACQUES LACAN ET FERNAND DELIGNY ${ }^{1}$
}

\author{
FRANCIELLI CRISTINA GIACOMINI
}

Maître en psychanalyse à Paris 8 Vincennes Saint-Denis, Diplômée em Licence en Sciences de l'Éducation à Faculdade de Filosofia, Ciências e Letras. ORCID: https://orcid.org/0000-00034772-2476. E-mail: fc giacomini@yahoo.com.br

\section{Résumé}

Lorsqu'on parle de l'autisme on est vite confronté à la question du langage et de l'absence de la parole. Comment peut-on s'en passer sans la voie traditionnel du symbolique ? L'abord plus raisonnable serait d'effectuer une recherche à ce propos étudiant les raisons de cette absence, de ce « déficit » qui cause de différents dysfonctionnements à niveau social, de l'apprentissage, etc. réfléchissant sur des méthodes de la prise en charge de l'autiste qui donneraient de réponses à ce vide. Toutefois, nous avons pris le contresens de direction. Notre abord théorique est justement étudier deux méthodes clinique et clinic-éducationnelle de prise en charge de l'autiste développé par Lacan et Deligny qui ne travaillent pas sur le manque ou

\footnotetext{
${ }^{1}$ Ce projet de recherche est développé sur l'orientation de Fabienne HULAK, psychologue clinicienne, HDR et maître de conférence à l'Université Paris 8 Vincennes Saint-Denis.
} 
sur le défaut du langage mais ils prennent la réponse donné par le corps lui-même comme façon d'établir un bord qui permet un certain équilibre dans le monde chaotique de l'autiste. Pourquoi le corps est-il en premier plan dans notre recherche ? Par absence de parole et non du langage, c'est le corps de l'autiste qui permet d'établir un lien avec le monde extérieur. Notre recherche a pour objectif mener une étude sur le corps dans l'autisme dans l'approche clinique en sciences de l'éducation à partir des théories de Jacques Lacan et de Fernand Deligny. Cette démarche est donc d'examiner un sujet qui a été très peu exploité et qui n’a jamais été affronté directement ni systématisé, rendant notre recherche inédite. Cette recherche, qui s'inscrit dans une démarche qualitative, est organisée selon un double enjeu : d'un côté l'enjeu théorique développant les concepts apportés par la DSM et les lois concernant la prise en charge de l'autisme dans la sphère médicale et éducationnelle ; et également l'apport psychanalytique de Jacques Lacan concernant la constitution du corps chez l'autiste et ses modes de traitements aussi bien que les méthodes innovatrices de prise en charge clinic-éducationnelle de Fernand Deligny. De l'autre côté l'enjeu empirique : en France, nous nous proposons d'accompagner le travail clinique d'orientation lacanienne fait avec les autistes dans l'Association Main à l'Oreille tandis qu'au Brésil au sein d'une institution éducationnelle nous allons réfléchir à l'application des méthodes de la cartographie et lignes d'erre proposées par Deligny. Notre objectif est de réfléchir la prise en charge de l'autisme comme un moyen de révéler un langage là où il y a vacance de la parole. Les résultats partiels qui nous avons pu repérer c'est qu'il n'y a pas de méthode unique d'apprentissage, ni de recette, ni de routine possibles appliquée à l'autisme parce que dans le monde auquel il vit, le symbolique est réel et l'imaginaire ne trouve pas forme dans la relation spéculaire. Par conséquent, les meilleures inventions qui se révèlent les plus solides pour (mieux) répondre à la souffrance de l'autiste sont celles du sujet lui-même.

Mots clés: autisme, corps, bord, lignes d'erres.

\section{O CORPO NO AUTISMO SEGUNDO JACQUES LACAN E FERNAND DELIGNY}

Quando se fala de autismo, somos rapidamente confrontados com a questão da linguagem e a ausência da fala. A abordagem mais convencional para realizar uma pesquisa seria tratar o assunto estudando as causas dessa ausência ou "déficit" que incitam diversas disfunções no nível social, da aprendizagem, etc. focando sobre os métodos clínico e de ensino que dariam respostas a essa falta. No entanto, priorizou-se a direção contrária, ou seja, na impossibilidade de passar pelo tradicional caminho do simbólico optouse estudar o corpo do autista, pois é ele que possibilita estabelecer uma ligação com o mundo exterior. À partir do estudo de dois métodos: o clínico e o clínico-educativo desenvolvidos por Lacan e Deligny respectivamente, pois seus enfoques não partem da falta ou da ausência da linguagem verbal, mas tomam a resposta dada pelo próprio corpo como uma forma de estabelecer um equilíbrio no mundo caótico do autista. O objetivo desta pesquisa é estudar sobre o corpo no autismo dentro de uma abordagem clínica em ciências da educação à partir das teorias de Jacques Lacan e Fernand Deligny. Essa aproximação teórica entre Lacan-Deligny nunca foi confrontada diretamente nem sistematizado, tornando esta pesquisa inédita. No que concerne a metodologia de pesquisa, este estudo estando inserido numa abordagem qualitativa e é organizado de acordo com um duplo enfoque: de um lado, a questão teórica que desenvolve os conceitos

\footnotetext{
p/Ulails Salvador, v. 3, n. 3, p.136-148, set./dez. 2018
}

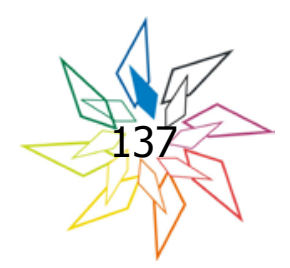


trazidos pelo DSM e as leis referentes ao tratamento do autismo na esfera médica e educacional; e também a contribuição psicanalítica de Jacques Lacan sobre a constituição do corpo no autismo e seus modos de tratamento, bem como os métodos inovadores de trabalho clínico-educacional de Fernand Deligny. De outro lado, a questão empírica: na França, propõe-se acompanhar o trabalho clínico de orientação lacaniana feito com autistas na Association Main à l'Oreille, enquanto que no Brasil propõe-se efetuar uma prática em uma instituição educacional para refletir sobre a aplicação dos métodos de cartografia e linhas de errância devenvolvidas por Deligny. Os resultados parciais que conseguimos identificar são, portanto, que não há um método único de aprendizado, nenhuma receita, nenhuma rotina possível aplicada ao autismo, porque no mundo em que ele vive, o simbólico é real e o imaginário não encontra forma na relação especular. Portanto, as melhores invenções que se mostram mais sólidas para (melhor) responder ao sofrimento dos autistas são as do próprio sujeito.

Palavras- chave: Autismo, corpo, bordas, linhas de errância.

\section{EL CUERPO EN EL AUTISMO SEGUN JACQUES LACAN Y FERNAND DELIGNY}

Cuando hablamos del autismo, nos enfrentamos rápidamente a la cuestión del lenguaje y la ausencia del mismo. ¿Cómo podemos hacer sin la forma simbólica tradicional? El enfoque más razonable sería llevar a cabo una investigación sobre este tema, estudiando las causas de esta ausencia, haciendo hincapé en métodos clínicos o de enseñanza que den respuestas a este vacío. Sin embargo, se prioriza la dirección contrária, esto es, ante la imosibidad de pasar por el tradicional camino del simbolismo, se opta por estudiar el cuerpo del autista, pues este es el que permite establecer una conexión con el mundo externo. Partiendo del estudio de dos métodos: el método clínico y el clínico-educativo desarrollados por Lacan y Deligny respectivamente, pues sus enfoques no parten de la falta o ausencia del lenguaje verbal, sino toman la respuesta dada por el propio cuerpo como una manera de establecer un equilibrio en el mundo caótico del autista Nuestra investigación tiene como objetivo realizar un estudio sobre el cuerpo en el autismo dentro de un abordage clínico en la ciencia de la educación a partir de las teorías de Jacques Lacan y Fernand Deligny. Por lo tanto, este enfoque es examinar un tema que ha sido poco explotado y nunca ha sido confrontado directamente o sistematizado, lo que hace que nuestra investigación no tenga precedentes. En lo que respecta a metodología de investigación, este estudio forma parte de un enfoque cualitativo y se organiza de acuerdo con un doble desafío: por un lado, la cuestión teórica que desarrolla los conceptos presentados por el DSM y las leyes sobre el manejo del autismo en la esfera médica. y educativo; y también la contribución psicoanalítica de Jacques Lacan con respecto a la constitución del cuerpo en el autismo y sus modos de tratamiento, así como los métodos innovadores de manejo clínico-educativo de Fernand Deligny. Por otro lado, la cuestión empírica: en Francia, se propone acompañar el trabajo clínico de orientación lacaniana realizado con autistas en la Asociación Main à l'Oreille, mientras que en Brasil se propone efectuar la practica en una institución educacional para reflexionar sobre la aplicación de los métodos de cartografía líneas de errancia desarrolladas por Deligny. Los resultados parciales que hemos podido identificar es que no hay un método único de aprendizaje, ninguna receta, ninguna rutina posible aplicada al autismo porque en el mundo que vive, lo simbólico es real y lo imaginario no encuentra forma en la relación especular. Por lo tanto, los mejores inventos que resultan ser los más sólidos para responder mejor al sufrimiento de los autistas son los del sujeto mismo.

Palabras clave, autismo, cuerpo, arista, líneas de erracia.

p/ullais Salvador, v. 3, n. 3, p. 136-148, set./dez. 2018 


\section{LE CORPS DANS LAUTISME SELON JACQUES LACAN ET FERNAND DELIGNY}

\section{Problematique generale ${ }^{2}$}

L'étude du corps permet de réfléchir à propos de sa constitution, de ce qu'il serait de l'ordre du réel, de la réalité, de l'image, du symbolique et du langage. Dans l'objectif d'éclairer la constitution du corps autistique, notre hypothèse est que la construction d'une approche théorique entre Fernand Deligny et Jacques Lacan est envisageable ; effet, en dépit de leurs propositions et méthodes parfois antinomiques, ils maintiennent un fil conducteur assez proche. En nous fondant sur cette approche Lacan-Deligny, qui jusqu'à aujourd'hui n'a pas été mise en relief, nous nous proposons de réfléchir au travail avec les autistes et à leur prise en charge thérapeutique et éducationnelle.

La prise de conscience de soi, de son corps, distinct des autres corps, a été objet de réflexion par les psychologues dans les années 1930. Henri Wallon (2009) conçoit le stade du miroir comme une étape clé de la constitution du moi. À partir de l'image extériorisé au miroir, l'unification de son corps surgit, qui jusqu'à lors n'avait été aperçu qu'en parties, les mains, les pieds.

Fernand Deligny (1999, p. 756), reprenant ce développement du stade du miroir, souligne davantage l'aspect de la confrontation de l'individu à des situations et à son milieu. Il reconnaît qu'il y a, d'un côté, le stade du miroir dont la reconnaissance du moi met en évidence le sujet et la parole et, de l'autre, la main, cette partie du corps qui est la première présence proche et permanente. D'après Deligny cette extension du corps portant une « mine inexploitée » apparaît ainsi comme la plus importante piste thérapeutique. Toutefois, pour qu'on puisse faire usage de cette main, un minimum d'unification de son corps est nécessaire

Jacques Lacan (1949/1966), partant du développement de Wallon, conçoit le stade du miroir comme formateur de la fonction du Je, dans le sens où « je » est distinct de l'autre. En tant que

\footnotetext{
2 Je tiens à remercier vivement Monsieur Marlon Miguel et le professeur Fabio Libório Rocha qui m'ont conseillé et qui ont relu lors de la rédation de ce projet. Je remercie également la professeur Sonia Matos pour son soutien et sa lecture critique d'achévement de ce projet.
}

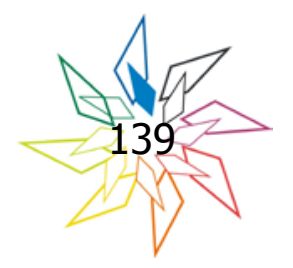


sujet social, il a besoin de l'Autre pour se constituer. Lacan introduit le rôle de l'Autre dans cette expérience de l'enfant. L'Autre apporte son regard et sa parole en désignant que l'image au miroir « c'est toi » et en permettant ainsi la reconnaissance imaginaire et l'acquisition de la fonction narcissique. C'est le moment où l'enfant se constitue par un autre spéculaire dans un processus d'aliénation à son semblable, celui du miroir, et son corps morcelé se constitue en imago du corps propre dans une totalité. C'est la jubilation de contempler son unité corporelle à partir de l'image spéculaire. En d'autres termes, l'enfant reconnaît d'abord l'image d'un autre pour seulement ensuite assumer cette image comme la sienne. C'est au stade du miroir que le corps scopique des pulsions libidinales est pris dans le champ visuel.

Avant que l'enfant puisse faire face à son image spéculaire, il ressent confusément des pulsions. Dans son champ visuel, il voit des morceaux de son corps. Cette construction permet de marquer l'indépendance de l'ordre imaginaire coupé du symbolique, parce que, selon Maurice Merleau-Ponty expliquant le développement de Lacan sur le stade du miroir, " je quitte la réalité de mon moi vécu pour me référer constamment à ce moi idéal, fictif ou imaginaire, dont l'image spéculaire est la première ébauche $»^{3}$. En d'autres mots, l'image spéculaire constitue le moi idéal qui sert de mode à la constitution du moi du sujet, dans un moi idéalisation au registre imaginaire. La figure de l'Autre, qui introduit son regard et sa parole devant le miroir, permet que l'enfant fasse des identifications au registre symbolique, instaurant son Idél du moi comme instance du discours et base des futurs choix objectaux, d'investissement libidinal, lié au non au narcissisme. Cette constitution de l'image du corps permettra de penser la question de la structure psychique du sujet.

Que se passe-t-il si cette constitution échoue ? Faute d'une enveloppe corporelle, le sujet ne réagit pas à l'image de son corps. On repère alors une indistinction entre l'enfant et l'autre, de sorte que la difficulté consiste à établir une délimitation entre le monde du sujet et celui de l'autre. Là où il n'y a pas d'Autre ni d'image spéculaire, d'après Hulak (2013), l'enfant est immergé dans la dimension du réel sans la mise en jeu de l'imaginaire, ni du symbolique.

Cette explication sur la constitution du corps amène à la réflexion concernant la constitution du corps dans l'autisme. Il semble que les autistes sont en deçà du miroir. Ils rencontrent une très grande difficulté, voire l'impossibilité, à se situer dans l'espace, et donc à installer des limites dans un monde chaotique.

\footnotetext{
${ }^{3}$ Extrait d'un cours de la Sorbonne ne possédant une date précise sur le site.
} 
Fabienne Hulak, dans son article « Le procès de subjectivation chez l'autiste » (2013), s'appuie sur des cas paradigmatiques pour expliquer le mode de subjectivation chez l'autiste. D'après Hulak, l'autiste entretient une relation spéciale à l'objet et au trou qui préfigure un abord topologique du problème de la subjectivation chez l'autiste. Elle affirme qu'à partir d'une relation transférentielle, l'autiste s'engage dans l'exploration de l'espace dans une invention de topologie en acte qui permet de border ce trou. Cette invention, apportée de soi-même par l'autiste, donne consistance au bord qui fait frontière entre un dehors et un dedans. Jacques-Alain Miller (2007) ${ }^{4}$ affirme que ce processus est en quelque sorte l'inverse de la symbolisation traduisant une réellisation du symbolique. Il s'agit, d'après Hulak, d'un effort de symbolisation avec des objets réels lié à l'imaginaire. C'est ce que Hulak appelle le réel de l'imaginaire dont la fonction est d'ouvrir à l'invention des formes d'accroche à la chaine signifiante. L'enfant, s'efforçant de s'y retrouver dans ce monde, va construire des dispositifs pour établir des frontières, de bords ou des contours dans l'espace. C'est l'invention d'une topologie en acte.

Le concept de «bord » a été précisé par Lacan dans son séminaire L'objet de la psychanalyse ${ }^{5}$, comme une limite ou une frontière, mais qui ne détermine ni intérieur ni extérieur. C'est aux frontières, seuils, limites, marges, bords que les enfants autistes doivent faire face.

Faute de construction structurale des bords, l'autiste est conduit à créer des frontières entre le soi et le non-soi à partir d'objets concrets comme despapiers, dessins, traces, nourriture, ou son propre corps qui délimite les frontières dans ses éplacements en surface ou en bordure dtrottoirs dans un comportement de frontières. Ce fait met en relief le lien du corpset de ses expériences sensorielles. fonction de ce comportement est l'apaisement de l'autiste de façon à rendre son monde plus vivable où une certaine dynamique libidinale est repérable. Cette libido, qui ultérieurement a été traduite par Lacan comme « jouissance », joue un rôle fondamental dans son rapport au corps et dans sa localisation sur ces bords.

Hulak (2013) affirme qu'en matière de psychanalyse, il n'y a pas de méthode d'apprentissage, ni de recette, ni de routine possibles appliquée à l'autisme. Ce qui nous amène à l'incontournable réflexion à propos de la prise en charge par les professionnels des équipes éducationnelles et thérapeutiques. Doit-on proposer ou apporter des objets sur lesquels l'autiste pourrait prendre appui de façon à maîtriser ses tensions internes? D'après la méthode de travail créée par Deligny pour la prise en charge de l'autiste, les cartes, par exemple, visent à diminuer

\footnotetext{
${ }^{4}$ Cité par Fabienne Hudans son article « Le procès de subjectivation chez l'autiste ».

${ }^{5}$ Séminaire inédit, sans publication actuel.
}

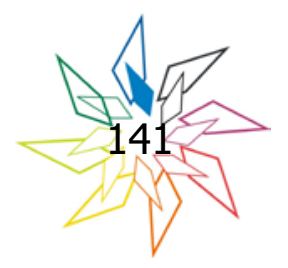


l'angoisse des soignants, mais aussi à établir un bord dans le comportement autistique. Cette méthode delignienne n'incite pas à cadrer de façon rigide une méthode éducationnelle, mais au contraire, elle répond autrement à l'appel du langage. Et du côté thérapeutique, d'après JacquesAlain Miller dans son texte "L'invention psychotique » (2004), les meilleures inventions qui se révèlent les plus solides pour (mieux) répondre à la souffrance du sujet sont celles du sujet luimême. C'est sur ces bases que la recherche prendra son essor.

\section{Etat de la recherche}

On constate depuis la publication Euvres, de Fernand Deligny, en 2007, une redécouverte de cet auteur et de son œuvre. En France, cet intérêt peut être attribué en partie à la publication inédite de certains de ses textes. Au Brésil, sa découverte se fait progressivement étant donné que les recherches sur ses œuvres sont très récentes et que les publications et le thèses à son sujet sont rares. Au contraire, les recherches sur la théorie de Lacan au Brésil sont très nombreuses. Cela est dû à l'intérêt croissant pour la psychanalyse dans le milieu académique et clinique.

Lorsqu'on analyse la bibliographie actuellement existante sur le corps dans l'autisme, on constate une absence de réflexion systématique et sérieuse dans l'approche transversale entre Lacan et Deligny. Les textes qui se rapportent au débat Lacan-Delingy sont réduits et, pour l'essentiel, cette recherche est inédite. Parmi les travaux actuellement existants, nous en évoquerons ici certains qui sont plus au moins liés à notre objet de recherche, à savoir, le corps autistique à partir de l'approche théorique de Lacan et Deligny.

L'approche théorique entre Lacan et Deligny a été esquissée dans la thèse de Marlon Miguel, À la marge et hors-champ : l'humain dans la pensée de Fernand Deligny (2016). Il a établi les interlocutions plus ou moins directes entre la pensée de Deligny et celle de Lacan. Sa thèse mettant au centre de ses réflexions l'humain dans la pensée de Fernand Deligny, Miguel (2016) mentionne que Deligny ne cesse de reprendre les définitions psychanalytiques, mobilisant notamment le vocabulaire lacanien pour parler de l'autisme et de la psychose en général. Son objectif est, selon l'auteur, que sa théorie puisse résonner dans le champ discursif clinique et que sa pratique puisse s'y inscrire. Miguel (2016) signale qu'en dépit des critiques de Deligny à propos du discours psychanalytique concernant surtout l'usage abusif de la parole et de l'interprétation, elles sont formulées notamment pour mieux souligner la différence et la singularité de son développement dans les Cévennes. Deligny a lu transversalement certaines œuvres de Lacan pour 
aussitôt y opposer ses propositions ou en incorporer quelques-unes. Néanmoins, sa lecture reste très partielle. Miguel (2016) affirme que Deligny avait probablement lu d'autres textes lacaniens au-delà de ceux qui ont été cités dans sa thèse; toutefois, il n'a pas pu en repérer les traces. Le travail reste encore à faire.

Fernand Deligny, Euvres, a été la source d'inspiration de l'article d'Annie Tardits (2008). L'auteur y développe la ou les position(s) de Fernand Deligny, notamment sa relation avec la psychanalyse et la complexité de son rapport à l'œuvre de Lacan. L'article évoque la lecture très sélective qu'a fait Deligny des œuvres de Lacan. Tardits (2008) évoque certains points intéressants de cette position delignienne par rapport aux développements lacaniens en ce qui concerne le langage. Deligny refuse la notion d'un prélangage ou d'un langage pré-verbal, en affirmant que nous n'avons pas de langage, mais il reconnaît le pouvoir du symbolique. En d'autres termes, Deligny reconnaît que nous sommes faits de langage, que « cette parole fait ce que nous sommes et qui règne, universelle, historique, démonstrative, cocasse, meurtrière » (TARDITS, 2008, p. 217). Ce qui fait que nous sommes «parasités " ${ }^{6}$ par le langage.

D'après Tardits (2008), Deligny soutient son argument en piochant dans les explications de Lacan dans son premier Séminaire quand il explique, à partir de l'exemple du mot éléphant, que le concept peut remplacer la chose. Ce propos lacanien ne passe pas inaperçu pour Deligny qui réfléchit à propos du concept de réel : ce réel «dont l'autiste ne se distingue pas » (TARDITS, 2008, p. 218). Deligny avance sur la voie de l'assujettissement au langage à sa façon particulière. Vis-à-vis de l'absence du langage verbal chez les autistes, Deligny inscrit son développement au seuil, au point de bifurcation où a eu lieu la « greffe » du langage et le «tracer d'avant la lettre».

L'approche Deligny-Lacan dans cet article valorise ce qui Deligny a pioché dans le développement de Lacan, ce qui fait qu'il reste très partiel à l'égard du développement théorique de Lacan. Il serait envisageable de lire Deligny àpartir de la théorie de Lacan afin de mettre en tension leurs concepts épistémologiques, de façon à apporter leur juste portée aux développements conceptuels des auteurs, leur conférant ainsi une approche vraisemblablement enrichissante.

Au Brésil, dans le domaine de Sciences de l'Éducation, au sujet de Deligny, nous citerons la thèse de 2016 de Julia de Jesus, Trajetórias e caminhos : uma cartografia dos bebês qui porte sur

\footnotetext{
${ }^{6}$ Terme lacanien développé dans son texte « Joyce et les paroles imposées ».
}

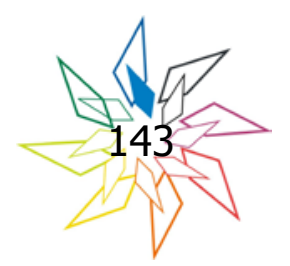


la cartographie des bébés comme façon d'y inscrire leurs trajectoires et leurs cheminements. Nous citerons aussi la thèse de 2014 de Sonia Matos, intitulé Procedimentos de escritura e afectologia na alfabetização de crianças : abordages cruzadas entre a filosofia da diferença e a psicologia intercultural qui consacre un chapitre aux cartes et lignes d'erre de Fernand Deligny. Matos décrit cette «méthode » d'expérimentation exploratoire de Deligny conformément aux explications de Deleuze et Guatari. Il s'agit d'une élaboration sémiotique de travail avec les autistes qui vivent dans la vacance du langage. Ses lignes d'erre sont considérées par les auteurs comme le pouvoir d'agir d'un corps qui fuit. Deligny ne parle pas de fuite ou d'évasion du corps car les lignes d'erre se reproduisent dans un espace grâce auquel elles peuvent s'y inscrire. Il ne s'agit pas, d'après lui, d'une manière de fuir. Cette méthode permet d'éloigner l'interprétation à partir du langage. Matos (2014) propose l'utilisation de cette méthode aux ateliers d'alphabétisation des enfants. La trace est tenue comme ligne d'erre ayant l'innocence comme aspect singulier de l'existence. Ce sont des tracés géographiquement placés sur une feuille qui ne veulent rien dire et qui ont le but d'agir.

En France, au sujet de Lacan et du corps autistique, nous citerons le travail de Marina Bialer, Le corps dans l'autisme et dans la schizophrénie, soutenue en 2012. Sa thèse porte sur la constitution du corps dans l'autisme et dans la schizophrénie à partir de deux concepts clés : l'objet a et le trait unaire. D'après Bialer (2012), l'autisme se caractérise par un défaut dans l'empreinte du trait unaire comme la trace de l'Autre symbolique qui permettrait l'inscription de l'érotisme, base de la construction narcissique, et de l'inscription de l'Autre au circuit pulsionnel. Ce défaut empêcherait le processus d'aliénation dans le signifiant de l'Autre et sa séparation postérieure. L'auteure part de la théorie lacanienne présentant l'approche psychanalytique de Jean-Claude Maleval qui privilégie le traitement de l'autisme à partir de la localisation de la jouissance sur le bord et également le traitement de l'autisme proposé par M.-C. Laznik qui défend la sortie de l'autisme à partir de l'inscription au champ du désir et du signifiant.

La thèse de Séverine Rézette, Autisme et représentations, datant de 2008, porte sur l'autisme et ses représentations. Le double enjeu des représentations est présenté par celles faites pour les psychologues qui travaillent avec les autistes et par celles auxquelles les autistes sont ou non capables d'accéder : les représentations du monde qui les entoure. La thèse, en présentant des cas cliniques d'adolescents autistes, questionne la structure autistique marquée par la présence/ absence de l'Autre afin de répondre à la problématique de comment l'autiste fait pour vivre.

Christian Degene (2015) a développé une thèse autour de l'abord psychanalytique sur la communication d'enfants pauvrement ou non verbalisant et visant à favoriser leur accession à la parole. Son objectif est de trouver des moyens analytiques de favoriser l'émergence du langage 
chez les enfants à risque d'évolution autistique. C'est une façon de répondre au troisième plan autisme sur l'interrogation de l'efficacité de la psychanalyse dans la prise en charge de l'autisme. D'après l'auteur, l'implication du corps se révèle être un événement charnière sur l'acquisition du langage. Il étudie la qualité des productions langagières et analyse les premiers sons émis par l'enfant afin de savoir s'il s'agit de vocalisation ou de verbalisation, de sens ou de non-sens. Il conclut que la tâche du psychanalyste est particulièrement empreinte d'incertitude et de tentative de décryptage.

\section{Presentation de la demarche et plan d'ensemble}

L'objectif de notre démarche est donc d'examiner un sujet qui a été très peu exploité et qui n'a jamais été affronté directement ni systématisé : nous nous proposons de mener une enquête sur le corps dans l'autisme dans l'approche clinique en sciences de l'éducation et vice-versa dans les théories de Jacques Lacan et de Fernand Deligny.

Cette recherche, qui s'inscrit dans une démarche qualitative, est organisée selon un double enjeu, l'enjeu théorique et l'enjeu empirique. D'une part, notre objectif est d'approfondir les connaissances sur l'autisme, son rapport au corps, son diagnostic et ses modes de traitement. On mettra en relief l'approche théorique de Lacan et Deligny afin de réfléchir sur ses méthodes et d'enrichir la pratique clinique-éducationnelle. D'autre part, notre objectif est d'accompagner le travail clinique d'orientation lacanienne fait avec les autistes dans l'Association Main à l'Oreille et le travail clinico-éducationnel par une approche delignienne.

L'axe théorique sera présenté en trois parties. D'abord, la recherche prendra appui sur les concepts développés dans le champ médical à partir du Manuel diagnostique et statistique des troubles mentaux (DSM) en ce qui concerne la définition, les symptômes, le diagnostic et les modes de traitements de l'autisme. Puis nous approfondirons les champs juridique et éducationnel concernant la prise en charge de l'autisme à sphère médicale et éducationnelle, dans le contexte actuel des lois à propos de l'autisme, d'une part, en France, et, d'autre part, au Brésil. Ensuite, nous souhaitons développer les apports de Jacques Lacan concernant la constitution du corps chez l'autiste et ses modes de traitements. Et enfin, nous voudrions présenter les apports de Fernand Deligny concernant le corps de l'autiste et ses méthodes innovatrices de prise en charge clinicéducationnelle. 
L'axe empirique sera présentée en deux parties. D'une part, en France, nous souhaitons accompagner le travail clinique d'orientation lacanienne fait avec les autistes dans l'Association Main à l'Oreille qui nous a d'ores et déjà donné son accord. Cette pratique nous permettra d'étudier de près l'aspect transférentiel de l'analyse et le travail autour des bords. Et d'autre part, pendant notre période en cotutelle au Brésil, nous enquêterons sur les dispositifs mis en place au sein de l'institution éducationnelle dans l'objectif de réfléchir à l'application des méthodes de la cartographie et lignes d'erre proposées par Deligny comme un moyen de révéler un langage là où il y a vacance de la parole. Son apport rompt avec les méthodes comportementalistes.

Cet étude nous permettra de faire l'approche clinique en sciences de l'éducation et viceversa de la méthode pédagogique/éducationnelle en clinique. Qu'apporte-on de la clinique en sciences de l'éducation? Notre analyse procédera par l'identification de cette présence de l'analyste, du soignant et de l'éducateur où l'enjeu transférentiel sera mis au point. Dans la perspective de Deligny, il s'agit d'une « présence proche » auprès des autistes, sans forçage; et, rejoignant Lacan, il situe le savoir du côté des autistes/patients afin de s'en laisser enseigner. Le point de convergence des deux méthodes est de mettre en lumière les inventions construites de soi-même par les autistes. Les résultats de cette recherche seront publiés dans un livre.

Dans le cadre d'une telle conception de recherche, l'essentiel dans l'autisme est de considérer sa position subjective qui relève d'un choix du sujet en l'écartant de sa dimension pathologique fixée et irréversible. Il sera introduit la dimension du sujet en évoquant sa responsabilité subjective valorisant l'émancipation humaine.

\section{BIBLIOGRAPHIE}

BIALER, M. Le corps dans l'autisme et dans la schizophrénie. Thèse de Doctorat, Université Paris 7 Diderot : Paris, 2012.

CARDOSO PINTO MIGUEL, Marlon. À la marge et hors-champ : l'humain dans la pensée de Fernand Deligny. Thèse de Doctorat, Université Paris 8 Vincennes Saint-Denis : Paris, 2016.

DA LUZ MATOS, Sonia Regina, Procedimentos de escritura e afectologia na alfabetização de crianças : abordages cruzadas entre a filosofia da diferença e a psicologia intercultural. Tese de Doutorado : Porto Alegre, 2014. 
DELIGNY, Fernand, Cartes et lignes d'erre. Traces du réseau de Fernand Deligny, Paris : L’Arachnéen, 2013.

, Nous et l'innocent : In : CEuvres, Paris : L’Arachnéen, 2007.

, Euvres, Paris : L’Arachnéen, 2007.

DEGENE, Christian, L'abord psychanalytique de communications d'enfants pauvrement ou non verbalisant, à visée de favoriser leur accession à la parole : l'existence d'une subjectivité purement autistique? Thèse de doctorat : Université Paris 7 Diderot, 2015.

HULAK,Fabienne. « Le procès de subjectivation chez l'autiste. D'une topologie en acte au problème de l'accroche à la chaîne signifiante ", Psychologie Clinique, Paris,n. 36, p. 92-102, fev. 2013.

JESUS, Julia de, Trajetórias e caminhos : uma cartografia dos bebês. Tese de doutorado : Universidade Federal de São Carlos, 2016.

LACAN, Jaques, « Joyce et les paroles imposées » in : Séminaire XXIII : Le sinthome, Paris : Seuil, p. 91-102, Séance Leçon du 17 février 1975.

, Le Séminaire XIII, L’objet de la psychanalyse. Inédit. Leçon du 5 janvier 1966.

, Le stade du miroir. In : Écrits I. Édition de poche. Paris : Seuil, 1966. p. 92-99. 1949

MERLEAU-PONTY, Maurice, Les relations à autrui chez l'enfant, éd. Les cours de la Sorbonne, pp.55-57. Disponible sur : http://lewebpedagogique.com/philosophie-bac/le-stade-du-miroirselon-lacan-texte-de-merleau-ponty/http://lewebpedagogique.com/philosophie-bac/le-stade-dumiroir-selon-lacan-texte-de-merleau-ponty/http://lewebpedagogique.com/philosophie-bac/lestade-du-miroir-selon-lacan-texte-de-merleau-ponty/ . Date d'acc: 19 mai 2018.

MILLER, Jacques-Alain, " La matrice du traitement de l'enfant aux loups », In : La cause freudienne, $\mathrm{n}^{\circ} 66$, mai 2007.

, « L'invention psychotique », In : Quarto, n80, janvier 2004.

RÉZETTE, Séverine, Autisme et représentations. Thèse de doctorat : Université Paris 7 Diderot, Paris 2008. 
TARDITS, Annie, Position(s) de Fernand Deligny : à propos du livre Fernand Deligny, Euvres, In: Essaim, Eres, n. 20, p. 213-220, jav. 2008. Disponible sur : https://www.cairn.info/revueessaim-2008-1-page-213.htm\#re1nolhttps://www.cairn.info/revue-essaim-2008-1-page-213. htm\#re1nolhttps://www.cairn.info/revue-essaim-2008-1-page-213.htm\#relnolhttps://www. cairn.info/revue-essaim-2008-1-page-213.htm\#relno1 , Date d'accès : 5 mai 2018.

WALLON, Henri, Les origines du caractère chez l'enfant. 6 ed. Paris : PUF, 2009. 